\title{
Intravitreal Aflibercept as Rescue Therapy for Post-Radiation Cystoid Macular Edema Resistant to Intravitreal Bevacizumab: Outcomes at 1 Year
}

\author{
Mohammed Ali Khan ${ }^{\mathrm{a}, \mathrm{b}} \quad$ Arman Mashayekhi $^{\mathrm{a}} \quad$ Jerry A. Shields ${ }^{\mathrm{a}}$ \\ Carol L. Shields ${ }^{\mathrm{a}}$ \\ ${ }^{\mathrm{a}}$ Ocular Oncology Service and ${ }^{\mathrm{b}}$ Retina Service, Wills Eye Hospital, Philadelphia, PA, USA
}

\section{Keywords}

Tumor · Choroidal melanoma $\cdot$ Radiotherapy · Cystoid macular edema $\cdot$ Anti-VEGF

\section{Abstract}

Background/Aims: To investigate the efficacy of intravitreal aflibercept as rescue therapy for post-radiation cystoid macular edema (CME) resistant to prior treatment with intravitreal bevacizumab (IVB). Methods: Retrospective, interventional, case-controlled series. Eyes with persistent post-radiation CME were treated with intravitreal aflibercept (2 $\mathrm{mg} / 0.05 \mathrm{~mL}$ ). Central macular thickness (CMT) and visual acuity were compared to a matched control group treated with only IVB at 1 year. Results: Ten eyes of 10 patients were included, with 5 eyes in the intervention and 5 in the control group. The eyes in the intervention group had previously been treated with IVB (mean 11.6 injections, range 6-22) but failed to show resolution of CME. Following rescue treatment with a mean of 9 injections of aflibercept, the mean CMT was reduced from $463 \pm 138$ to $267 \pm 80 \mu \mathrm{m}(p=0.02)$ and the mean Snellen visual acuity was improved from 20/67 to $20 / 42(p=0.03)$. At 1 year, the eyes in the intervention group had lower CMT (267 \pm 80 vs. $361 \pm 71 \mu \mathrm{m}, p=0.09)$ and significantly better Snellen visual acuity (20/48 vs. $20 / 76, p=$

\section{KARGER}

(C) 2017 S. Karger AG, Basel

E-Mail karger@karger.com

www.karger.com/oop
0.02) compared to the control group. Conclusions: Aflibercept may be an effective rescue therapy for persistent postradiation $C M E$ in eyes with incomplete response to IVB, with reduction in CMT and improvement in visual acuity.

(c) 2017 S. Karger AG, Basel

\section{Introduction}

Post-radiation cystoid macular edema (CME) is a common cause of vision loss following plaque radiotherapy for uveal melanoma $[1,2]$, occurring in up to $70 \%$ of eyes after iodine-125 plaque radiotherapy at 2 years [3]. Prior studies have identified many factors that are associated with the development of post-radiation CME including larger tumor size, higher radiation dose, and increased central macular thickness (CMT) at presentation $[3,4]$, which may help clinicians identify patients at higher risk.

As both uveal melanoma and ischemic sequelae of radiotherapy elevate levels of VEGF [5], intravitreal antiVEGF agents have been commonly utilized for patients with post-radiation CME [6-8]. Multiple reports have noted improvement in visual acuity and CMT in eyes with post-radiation CME treated with intravitreal bevacizumab (IVB) [7, 9]; however, a subset of patients will de-

Arman Mashayekhi, MD

Ocular Oncology Service, Wills Eye Hospital

840 Walnut Street, Suite 1440

Philadelphia, PA 19107 (USA)

E-Mail arman_mash@yahoo.com 
Table 1. Intravitreal aflibercept for postradiation cystoid macular edema resistant to intravitreal bevacizumab: baseline ocular and tumor features

\begin{tabular}{|c|c|c|c|}
\hline Feature & $\begin{array}{l}\text { Intervention group } \\
(n=5 \text { eyes })\end{array}$ & $\begin{array}{l}\text { Control group } \\
\text { ( } n=5 \text { eyes })\end{array}$ & $p$ value \\
\hline Age, years & $62.6(62,57-69)$ & $56(58,41-70)$ & 0.40 \\
\hline \multicolumn{4}{|l|}{ Gender } \\
\hline Female & 5 & 4 & \\
\hline Male & 0 & 1 & \\
\hline \multicolumn{4}{|l|}{ Race } \\
\hline Caucasian & 5 & 5 & \\
\hline \multicolumn{4}{|l|}{ Ocular features } \\
\hline Choroidal melanoma & 5 & 5 & \\
\hline Lens crystalline & 5 & 5 & \\
\hline Epiretinal membrane & 0 & 0 & \\
\hline Fluorescein angiography-evident cystoid macular edema & 0 & 0 & \\
\hline Optical coherence tomography-evident cystoid macular edema & 0 & 0 & \\
\hline \multicolumn{4}{|l|}{$\log M A R$ visual acuity } \\
\hline Affected eye & $0.19(0.18,0.10-0.3)$ & $0.15(0,0-0.60)$ & 0.73 \\
\hline \multicolumn{4}{|l|}{ Central macular thickness, $\mu \mathrm{m}$} \\
\hline Affected eye & $315.2(272,252-489)$ & $329(283,247-552)$ & 0.53 \\
\hline Fellow eye & $267(255,232-302)$ & $266(266,248-281)$ & 0.91 \\
\hline \multicolumn{4}{|l|}{ Tumor dimensions, $\mathrm{mm}$} \\
\hline Basal diameter & $10.7(10,6-16)$ & $10.9(11,7-18)$ & 0.88 \\
\hline Thickness & $3.6(3.6,2.2-4.7)$ & $4.6(3.9,2.5-9.6)$ & 0.58 \\
\hline Distance to foveola & $4.3(3,0.5-12)$ & $4.9(2.5,2-15)$ & 0.89 \\
\hline Distance to optic nerve & $5.4(6,3-9)$ & $5(5,0.5-12)$ & 0.90 \\
\hline
\end{tabular}

Figures are numbers or means with medians and ranges in parentheses.

velop CME that is persistent despite a fixed-dose monthly therapy [7]. Rescue therapies for such patients with recalcitrant post-radiation CME have not been well described to date.

Aflibercept (Eylea; Regeneron Pharmaceuticals, Inc.) is a fully human, soluble recombinant VEGF receptor decoy that inhibits VEGF isoforms A and B (VEGF-A, VEGF-B) and placental growth factor (PlGF). Preclinical studies have shown that the binding affinity of aflibercept for VEGF-A is higher than native VEGF receptors [10]. The purpose of this study was to assess the efficacy of intravitreal aflibercept as rescue therapy for persistent postradiation CME resistant to prior treatment with IVB.

\section{Methods}

Institutional Review Board approval from Wills Eye Hospital was obtained for this retrospective, interventional, case-controlled series. This research adhered to the tenets of the Declaration of
Helsinki and was conducted in accordance with regulations set forth by the Health Insurance Portability and Accountability Act. All patients were managed at the Ocular Oncology Service of Wills Eye Hospital, Philadelphia, PA, USA.

The clinical records of all patients with persistent post-radiation CME following iodine-125 plaque radiotherapy for uveal melanoma treated with intravitreal aflibercept were reviewed. Persistent post-radiation CME was defined as increased or stable CMT compared to baseline values despite a minimum of 3 monthly standard-dose (1.25 mg) IVB treatments. For the purpose of this study, change in CMT between baseline and follow-up was classified as decreased ( $>10 \%$ decrease in CMT compared to baseline), stable (within $10 \%$ of baseline CMT), or increased ( $>10 \%$ increase in CMT compared to baseline).

The following demographic and clinical features were recorded: age, gender, lens status, past ocular and medical history, Snellen visual acuity, tumor location, tumor diameter, and tumor thickness. Parameters of iodine- 125 plaque radiotherapy, including total dose (cGy) and dose rate (cGy/h) to the tumor apex, base, optic disc, and foveola, were noted. Details regarding onset and treatment for post-radiation CME were also recorded.

Five eyes of 5 patients with persistent post-radiation CME were treated with aflibercept and included in the intervention group. All 
Table 2. Intravitreal aflibercept for postradiation cystoid macular edema resistant to intravitreal bevacizumab: radiation parameters

\begin{tabular}{|c|c|c|c|}
\hline Feature & $\begin{array}{l}\text { Intervention group } \\
(n=5 \text { eyes })\end{array}$ & $\begin{array}{l}\text { Control group } \\
(n=5 \text { eyes })\end{array}$ & $p$ value \\
\hline \multicolumn{4}{|l|}{ Radiation dose, cGy } \\
\hline Tumor apex & $7,200(7,000,7,000-8,000)$ & $7,600(8,000,7,000-8,000)$ & 0.37 \\
\hline Tumor base & $14,254(13,840,11,350-20,440)$ & $17,710(16,460,9840-24,200)$ & 0.36 \\
\hline Foveola & $6,158(3,170,1,581-13,001)$ & $3,745(3,949,2,560-4,184)$ & 0.39 \\
\hline Optic disc & $2,584(2,554,1,548-3,647)$ & $3,717(3,910,1,690-5,595)$ & 0.20 \\
\hline \multicolumn{4}{|l|}{ Radiation rate, $\mathrm{cGy} / \mathrm{h}$} \\
\hline Tumor apex & $74.1(74.7,59.2-84.8)$ & $76.7(81.7,60.2-85.9)$ & 0.75 \\
\hline Tumor base & $143.8(147.6,120.4-172.9)$ & $175.6(176.7,103.8-223.9)$ & 0.27 \\
\hline Foveola & $65.5(33.8,13.4-138.0)$ & $38.1(42.4,22.0-42.8)$ & 0.38 \\
\hline Optic disc & $26.6(25.5,16.5-38.7)$ & $37.4(33.6,17.8-60.1)$ & 0.30 \\
\hline \multicolumn{4}{|c|}{$\begin{array}{l}\text { Onset of optical coherence tomography-evident } \\
\text { cystoid macula edema }\end{array}$} \\
\hline Months following plaque & $22.8(14,8-43)$ & $25.6(16,9-61)$ & 0.73 \\
\hline logMAR visual acuity, affected eye & $0.47(0.48,0.40-0.60)$ & $0.60(0.60,0-0.88)$ & 0.47 \\
\hline CMT, affected eye, $\mu \mathrm{m}$ & $463.4(495,354-642)$ & $444.4(427,374-552)$ & 0.66 \\
\hline CMT, fellow eye, $\mu \mathrm{m}$ & $267(265,256-293)$ & $270(264,254-299)$ & 0.81 \\
\hline
\end{tabular}

Figures are means with medians and ranges in parentheses.

eyes were treated with a minimum of 6 monthly injections of intravitreal aflibercept, with follow-up examinations and spectral domain optical coherence tomography (OCT) performed every 4 weeks. After 6 months, the treatment interval could be adjusted based on clinical response. Outcomes at 6 months and 1 year were then compared with a matched control group comprised of 5 eyes of 5 patients with persistent post-radiation CME treated only with IVB. Controls were matched for baseline tumor features, CMT, visual acuity, the number of prior IVB treatments received, and follow-up interval.

Snellen visual acuities were converted to logMAR equivalents for statistical analysis. Using a Student $t$ test analysis (GraphPad Software Inc., La Jolla, CA, USA), a $p$ value $<0.05$ was considered statistically significant. The primary outcomes measured included change in CMT and visual acuity following treatment with aflibercept.

\section{Results}

Baseline features including patient age, gender, race, lens status, visual acuity, CMT in the affected and fellow eye, tumor thickness, tumor diameter, and tumor distance to the foveola were statistically similar between the intervention and control group (Table 1).

In the intervention group, mean $\log M A R$ visual acuity at the time of melanoma diagnosis was $0.19 \pm 0.10$ (Snellen 20/30). Mean tumor basal diameter was $10.7 \mathrm{~mm}$ (median 10, range 6-16) and mean tumor thickness was 3.6 mm (median 3.6, range 2.2-4.7). In the control group, mean $\log M A R$ visual acuity at the time of melanoma diagnosis was $0.15 \pm 0.26$ (Snellen 20/28). Mean tumor basal diameter was $10.9 \mathrm{~mm}$ (median 11 , range $7-18$ ) and mean tumor thickness was $4.6 \mathrm{~mm}$ (median 3.9, range 2.6-9.6). Angiographic or OCT-evident CME, macular ischemia, and epiretinal membrane were not present in any eye at baseline. All patients were phakic throughout the study period.

Details of iodine- 125 plaque radiotherapy are summarized in Table 2. In the intervention group, OCT-evident post-radiation CME occurred at a mean of 23 months (median 14, range 8-43). Mean CMT at diagnosis of postradiation CME was $479 \pm 123 \mu \mathrm{m}$ and mean logMAR visual acuity was $0.47 \pm 0.08$ (Snellen 20/60) in the affected eye. In the control group, OCT-evident post-radiation CME occurred at a mean of 26 months (median 16, range 9-61). Mean CMT at diagnosis of post-radiation CME was $444 \pm 71 \mu \mathrm{m}$ and mean $\log$ MAR visual acuity was $0.60 \pm 0.39$ (Snellen 20/79) in the affected eye.

Treatment outcomes are summarized in Table 3. In the intervention group, eyes were treated with a mean of 11.6 IVB injections (median 10, range 6-22) over a mean duration of 13 months (median 9, range 8-24). Following treatment with 6 monthly injections of intravitreal aflibercept, mean CMT improved from $463 \pm 138$ to 289 
Table 3. Intravitreal aflibercept for postradiation cystoid macular edema resistant to intravitreal bevacizumab: treatment outcomes

\begin{tabular}{|c|c|c|c|}
\hline Feature & Intervention group & Control group & $\begin{array}{l}\text { Comparison } \\
\text { between groups, } \\
p \text { value }\end{array}$ \\
\hline \multicolumn{4}{|l|}{ Prior cystoid macular edema treatment } \\
\hline Total prior intravitreal bevacizumab injections & $11.6(10,6-22)$ & $11(11,11-11)$ & 0.84 \\
\hline Treatment duration, months & $13.2(9,8-24)$ & $17.4(12.4,10.5-31)$ & 0.41 \\
\hline \multicolumn{4}{|l|}{ Baseline at initiation of rescue therapy } \\
\hline Central macular thickness, affected eye, $\mu \mathrm{m}$ & $463.4(407,329-682)$ & $406.8(413,288-568)$ & 0.60 \\
\hline Central macular thickness, fellow eye, $\mu \mathrm{m}$ & $262.6(254,221-293)$ & $279.4(275,252-307)$ & 0.44 \\
\hline $\log M A R$ visual acuity & $0.53(0.48,0.40-0.70)$ & $0.51(0.40,0.30-0.88)$ & 0.73 \\
\hline \multicolumn{4}{|l|}{ Outcomes at 6 months } \\
\hline Central macular thickness, affected eye, $\mu \mathrm{m}$ & $288.8(277,204-393)$ & $374.2(331,289-512)$ & 0.09 \\
\hline$p$ value & 0.04 & 0.66 & - \\
\hline $\log$ MAR visual acuity & $0.32(0.30,0.18-0.54)$ & $0.58(0.48,0.30-1.0)$ & 0.03 \\
\hline$p$ value & 0.03 & 0.20 & - \\
\hline \multicolumn{4}{|l|}{ Outcomes at 1 year } \\
\hline Number of rescue therapy injections & $9(8,8-11)$ & $10(10,9-11)$ & \\
\hline Central macular thickness, affected eye, $\mu \mathrm{m}$ & $267.4(268,193-387)$ & $361.2(331,282-457)$ & 0.09 \\
\hline$p$ value & 0.02 & 0.49 & - \\
\hline $\log M A R$ visual acuity & $0.32(0.40,0.0-0.54)$ & $0.58(0.54,0.40-0.88)$ & 0.02 \\
\hline$p$ value & 0.03 & 0.09 & - \\
\hline
\end{tabular}

Figures are means with medians and ranges in parentheses.

$\pm 68 \mu \mathrm{m}(p=0.04)$ and mean logMAR visual acuity improved from $0.53 \pm 0.15$ (Snellen 20/67) to $0.32 \pm 0.19$ (Snellen $20 / 42, p=0.03$ ). A statistically significant reduction in CMT was observed following only 2 aflibercept injections $(p=0.04)$, with an illustrative case depicted in Figure 1. At the mean final follow-up of 12.8 months (range 12-14), logMAR visual acuity remained stable at $0.32 \pm 0.23$ (Snellen 20/42, $p=0.03$ ) and CMT further improved to $267.4 \mu \mathrm{m}(p=0.02)$ following a mean of 10 (range 9-12) aflibercept injections. Following the 6-month time point, the treatment interval was extended in all patients to a mean of 8.6 weeks (range 6-13).

Compared to the control group, the eyes in the intervention group had comparatively lower CMT $(289 \pm 68$ vs. $374 \pm 94 \mu \mathrm{m}, p=0.10)$ and better visual acuity $(0.32$ \pm 0.19 vs. $0.56 \pm 0.28, p=0.03$ ) at 6 months. These differences were maintained at 1 year, with lower CMT $(289 \pm 70$ vs. $361 \pm 71 \mu \mathrm{m}, p=0.10)$ and better visual acuity $(0.38 \pm 0.21$ vs. $0.58 \pm 0.18, p=0.02)$ in the intervention group. There were no injection-related complications.

\section{Discussion}

Plaque radiotherapy is the standard of care for most small or medium-sized choroidal melanomas, providing excellent local tumor control [11]. However, vision loss is common following plaque radiotherapy. In a series of 1,106 patients, Shields et al. [2] reported visual acuity outcomes of $20 / 200$ or worse in up to $68 \%$ of patients treated with plaque radiotherapy for uveal melanoma at 10 years, with many of such outcomes attributed to sequelae of radiation retinopathy.

To better address visual acuity loss from CME specifically, recent work has sought to prevent occurrence of post-radiation CME as well as identify patients who may be at risk for CME development. Shah et al. [12] found that the use of IVB $(1.25 \mathrm{mg})$ at 4 -month intervals following plaque radiotherapy reduced the rate of OCT-evident CME (26 vs. $40 \%$ ) and moderate vision loss (15 vs. $28 \%$ ) in treated versus untreated eyes at 2 years, offering clinicians a possible preventative strategy. Moreover, a recent report identified that eyes with subclinical macular edema (greater than a $10-\mu \mathrm{m}$ difference in CMT between the affected and fellow eye) at baseline were more 
Fig. 1. Spectral domain optical coherence tomography of a 59-year-old female with post-iodine-125 plaque radiotherapy cystoid macular edema. While absent at diagnosis (a), cystoid macular edema developed 8 months following radiotherapy (b). After 10 intravitreal injections of bevacizumab (c), the edema persisted. Following treatment with aflibercept, the edema resolved as seen after 3 (d) and 5 (e) rescue treatments. CMT, central macular thickness; VA, visual acuity.

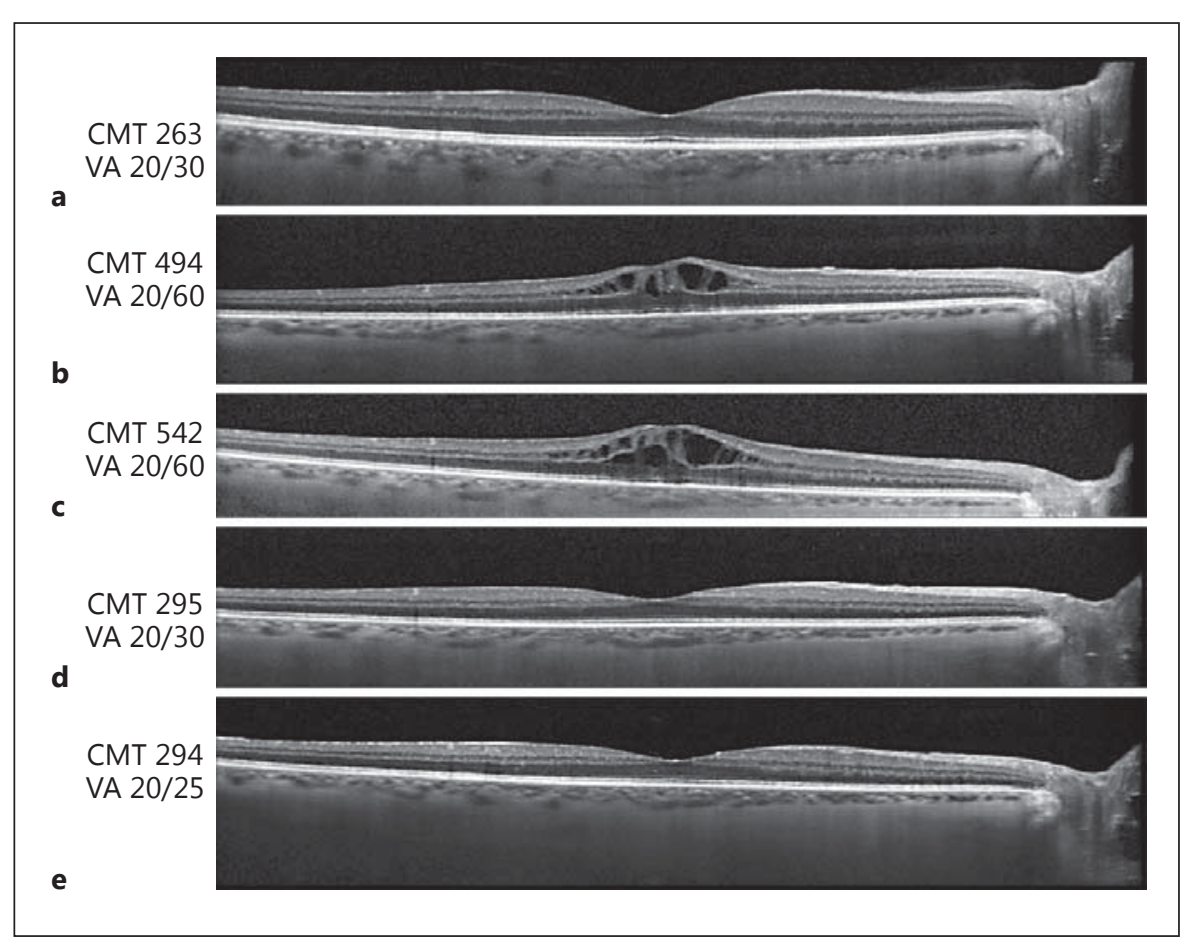

likely to develop CME after radiation ( $p=0.005$; hazard ratio 1.77), offering clinicians an OCT-based criterion to identify high-risk eyes [4].

Similarly, recent case series have sought to evaluate rescue therapies for persistent CME in eyes in which monthly anti-VEGF therapy fails to achieve CME resolution. Bui et al. [13] reported CMT and visual acuity outcomes in 2 patients with persistent post-radiation CME treated with the Ozurdex ${ }^{\circledR}$ dexamethasone implant (Allergan, Inc., Troy Hills, NJ, USA). The authors noted sustained anatomic improvement with Ozurdex ${ }^{\circledR}$ compared to prior IVB and intravitreal triamcinolone treatments, but visual acuity improved in only 1 of 2 eyes. Tarmann et al. [14] also reported outcomes in 4 eyes treated with Ozurdex ${ }^{\circledR}$ for persistent post-radiation CME despite prior IVB therapy. Similar to the results of Bui et al. [13], improvement in CMT was noted in all eyes but visual acuity improved in only 2 of 4 eyes. In addition, Finger and Chin [15] reported the efficacy of high-dose (2.0 $\mathrm{mg})$ ranibizumab in treating 10 patients' persistent radiation retinopathy despite monthly, standard-dose ranibizumab, hypothesizing that a higher-dose anti-VEGF blockade may improve treatment response in patients with recalcitrant disease. The authors noted that while $80 \%$ of the eyes had a statistically significant improvement in CMT at 1 year, visual acuity remained stable.

Aflibercept for Post-Radiation CME
The use of aflibercept for post-radiation CME is currently off-label. In this series, we found a statistically significant reduction in CMT $(p=0.02)$ and improvement in visual acuity ( $p=0.03$ ) following the use of monthly aflibercept at 6 months, gains that remained statistically significant at 1 year. In contrast, CMT did not improve significantly $(p=0.49)$ and visual acuity worsened ( $p=$ 0.09 ) in the control group at 1 year. Comparing groups, CMT was lower $(p=0.09)$ and visual acuity was significantly better $(p=0.02)$ in the aflibercept group at 1 year. Differing from the findings of Finger and Chin [15] evaluating high-dose ranibizumab therapy and the series evaluating Ozurdex ${ }^{\circledR}$ as rescue therapy for persistent post-radiation CME [13-15], statistically significant improvement in vision was observed with aflibercept treatment.

The precise mechanism for the observed reduction in CMT and improvement in visual acuity with aflibercept compared to IVB is speculative. Aflibercept has been demonstrated to bind VEGF-A with over 100-fold greater affinity than bevacizumab, possibly resulting in a more sustained VEGF-A inhibition [16-18]. Pharmacokinetic studies have also noted a longer predicted duration of action for aflibercept compared to bevacizumab and ranibizumab $[19,20]$. In addition, aflibercept inhibits not only VEGF-A but additional growth factors including

Ocul Oncol Pathol 2017;3:313-319 317 
VEGF-B and PlGF, which have been implicated in the pathogenesis of cystoid edema in other disease states including diabetic macular edema [16-18]. PlGF blockade may also have anti-inflammatory effects, as PIGF signaling has been implicated in monocyte chemotaxis and tissue factor (i.e., factor III, thromboplastin, CD142) production [21]. The higher affinity anti-VEGF blockade, longer duration of action, and simultaneous blockade of the PlGF cascade may all contribute to the visual acuity and CMT benefit noted with aflibercept in this series. Lastly, mechanisms of tachyphylaxis, including upregulation of VEGF receptors or metabolic changes altering pharmacokinetics of bevacizumab drug absorption, have been previously described with the use of anti-VEGF agents and could result in a dose-independent reduction in bevacizumab treatment response over time [22].

In the present study, exudative recurrences did not occur following treatment with aflibercept at a mean final follow-up of 12.8 months. In addition, all patients treated with aflibercept could be transitioned to a "treat and extend" regimen at 6 months, with treatment interval extension ranging from 6 to 13 weeks, potentially reducing treatment burden. Longer follow-up will be necessary to determine if the effect of aflibercept on CMT reduction and visual acuity reduction is maintained. A large cohort study has previously reported loss of at least 5 lines of Snellen visual acuity in up to $33 \%$ of patients undergoing plaque radiotherapy for uveal melanoma at 5 years [2]. Patients in the intervention group were followed for a mean of 45 months (3.7 years) from the time of plaque radiotherapy to the final follow-up. Over that time, mean logMAR visual acuity changed from 0.19 (Snellen 20/30) to 0.32 (Snellen 20/42), a loss of approximately 1 line of Snellen visual acuity. In the context of prior work noting significant visual loss following plaque radiotherapy in eyes with uveal melanoma, we believe the visual acuity results of this study are reflective of the improved outcomes that can be achieved after radiotherapy in the antiVEGF era.

This study has several limitations, including the retrospective nature and the small sample size. In addition, while no statistically significant differences between the intervention and control group were present at baseline, exact matching of a control group was not possible. In addition, follow-up fluorescein angiography after initiation of rescue therapy was not available in all patients, and thus subsequent development of macular ischemia and potential influence on visual acuity outcomes could not be evaluated. However, given the rarity of choroidal melanoma and paucity of information regarding rescue ther- apy for persistent CME, these results could be relevant and warrant future consideration. Additional studies, including the evaluation of aflibercept in treatment-naïve patients and prospective, randomized trials comparing treatment options would be necessary to clarify best practices.

In conclusion, intravitreal aflibercept was an effective rescue treatment for post-radiation CME in eyes with incomplete response to IVB. Statistically significant improvement in CMT and logMAR visual acuity was observed at 6 months that was maintained at 1-year. Additional follow-up and inclusion of a larger cohort is necessary to further define the role of aflibercept as rescue therapy for persistent post-radiation CME.

\section{Acknowledgements}

The authors did not receive grant support or research funding for this report.

\section{Statement of Ethics}

Institutional Review Board approval from Wills Eye Hospital was obtained for this study. This research adhered to the tenets of the Declaration of Helsinki and was conducted in accordance with regulations set forth by the Health Insurance Portability and Accountability Act.

\section{Disclosure Statement}

The authors do not have any proprietary interests in the materials described in the report. No conflicting relationship exists for any author. Arman Mashayekhi, MD has had full access to all the data in the study and takes responsibility for the integrity of the data and the accuracy of the data analysis.

\section{Author Contributions}

Design of the study: M.A.K., A.M., and C.L.S. Conduct of the study: M.A.K., A.M., C.L.S., and J.A.S. Data collection and analysis: M.A.K. and A.M. Interpretation of data: M.A.K., A.M., C.L.S., and J.A.S. Manuscript preparation: M.A.K., A.M., C.L.S., and J.A.S.
318

Ocul Oncol Pathol 2017;3:313-319

DOI: $10.1159 / 000452163$
Khan/Mashayekhi/Shields/Shields 


\section{References}

1 Horgan N, Shields CL, Mashayekhi A, et al: Classification and treatment of radiation maculopathy. Curr Opin Ophthalmol 2010; 21:233-238.

2 Shields CL, Shields JA, Cater J, et al: Plaque radiotherapy for uveal melanoma: long-term visual outcome in 1,106 consecutive patients. Arch Ophthalmol 2000;118:1219-1228.

3 Horgan N, Shields CL, Mashayekhi A, et al: Early macular morphological changes following plaque radiotherapy for uveal melanoma. Retina 2008;28:263-273.

4 Mashayekhi A, Schönbach E, Shields CL, et al: Early subclinical macular edema in eyes with uveal melanoma: association with future cystoid macular edema. Ophthalmology 2015; 122:1023-1029.

5 Missotten GSO, Notting IC, Schlingemann $\mathrm{RO}$, et al: Vascular endothelial growth factor a in eyes with uveal melanoma. Arch Ophthalmol 2006;124:1428-1434.

6 Finger PT, Chin KJ: Intravitreous ranibizumab (lucentis) for radiation maculopathy. Arch Ophthalmol 2010;128:249-252.

7 Mashayekhi A, Rojanaporn D, Al-Dahmash $S$, et al: Monthly intravitreal bevacizumab for macular edema after iodine- 125 plaque radiotherapy of uveal melanoma. Eur J Ophthalmol 2014;24:228-234.

8 Gupta A, Muecke JS: Treatment of radiation maculopathy with intravitreal injection of bevacizumab (Avastin). Retina 2008;28:964-968.
9 Reichstein D: Current treatments and preventive strategies for radiation retinopathy. Curr Opin Ophthalmol 2015;26:157-166.

10 Holash J, Davis S, Papadopoulos N, et al: VEGF-Trap: a VEGF blocker with potent antitumor effects. Proc Natl Acad Sci USA 2002; 99:11393-11398.

11 Jampol LM, Moy CS, Murray TG, et al: The COMS randomized trial of iodine 125 brachytherapy for choroidal melanoma: IV. Local treatment failure and enucleation in the first 5 years after brachytherapy. COMS report No 19. Ophthalmology 2002;109:2197-2206.

12 Shah SU, Shields CL, Bianciotto CG, et al: Intravitreal bevacizumab at 4-month intervals for prevention of macular edema after plaque radiotherapy of uveal melanoma. Ophthalmology 2014;121:269-275.

13 Bui KM, Chow CC, Mieler WF: Treatment of recalcitrant radiation maculopathy using intravitreal dexamethasone (Ozurdex) implant. Retin Cases Brief Rep 2014;8:167-170.

14 Tarmann L, Langmann G, Mayer C, et al: Ozurdex ${ }^{\circledR}$ reduces the retinal thickness in radiation maculopathy refractory to bevacizumab. Acta Ophthalmol 2014;92:e694-e696.

15 Finger PT, Chin KJ: High-dose (2.0 mg) intravitreal ranibizumab for recalcitrant radiation retinopathy. Eur J Ophthalmol 2013;23:850856.
16 Stewart MW, Rosenfeld PJ: Predicted biological activity of intravitreal VEGF Trap. Br J Ophthalmol 2008;92:667-668.

17 Stewart MW, Rosenfeld PJ, Penha FM, et al: Pharmacokinetic rationale for dosing every 2 weeks versus 4 weeks with intravitreal ranibizumab, bevacizumab, and aflibercept (vascular endothelial growth factor Trap-eye). Retina 2012;32:434-457.

18 Papadopoulos N, Martin J, Ruan Q, et al: Binding and neutralization of vascular endothelial growth factor (VEGF) and related ligands by VEGF Trap, ranibizumab and bevacizumab. Angiogenesis 2012;15:171-185.

19 Stewart MW, Rosenfeld PJ: Predicted biological activity of intravitreal VEGF Trap. Br J Ophthalmol 2008;92:667-668.

20 Stewart MW: Predicted biologic activity of intravitreal bevacizumab. Retina 2007;27:11961200.

21 Clauss M, Weich H, Breier G, et al: The vascular endothelial growth factor receptor Flt-1 mediates biological activities. Implications for a functional role of placenta growth factor in monocyte activation and chemotaxis. J Biol Chem 1996;271:17629-17634.

22 Gasperini JL, Fawzi AA, Khondkaryan A, et al: Bevacizumab and ranibizumab tachyphylaxis in the treatment of choroidal neovascularisation. Br J Ophthalmol 2012;96:14-20. 